\title{
A Malrotated, Incarcerated Appendix within an Epigastric Hernia
}

\author{
Patrick E. McGovern, MD ${ }^{1}$, Leandra Krowsoski, MD ${ }^{1}$ and Joseph F. Rappold, MD, FACS ${ }^{1,2^{*}}$
}

${ }^{1}$ Division of Trauma and Surgical Critical Care, Department of Surgery, Lewis Katz School of Medicine at Temple University, Philadelphia, USA

${ }^{2}$ Department of Surgery, Maine Medical Center, Portland, ME, USA

*Corresponding author: Dr. Joseph F. Rappold, MD, FACS, Department of Surgery, Maine Medical Center, Maine Health, 887 Congress Street, Suite 210, Portland, ME 04102, USA, Tel: 207-661-7081

\begin{abstract}
Background: A male patient with previously undiagnosed intestinal malrotation presented with symptomatic appendiceal incarceration within an epigastric hernia.

Summary: A 40-year-old male who presented with non-reducible ventral abdominal mass that was tender to palpation on physical examination. He was found to have appendiceal incarceration within an epigastric ventral hernia defect secondary to congenital malrotation of the bowel, as initially identified on computed tomography. The patient was taken to the operating room emergently, where reduction of the hernia sac, appendectomy, and proper rotation of the colon was performed. Pathologic findings were consistent with early inflammation within the appendix and a normal appearing hernia sac. Intestinal malrotation is an embryologic defect known to put patients at risk for serious complications, starting from birth and continuing throughout life. Though, typical management involves intervention in the neonatal and childhood periods, the rate and variety of complications that result from foregoing surgical correction in the absence of clinical symptoms is largely unknown. This case illustrates a potential complication associated with failure to correct both congenitally malrotated bowel and an epigastric hernia.
\end{abstract}

Conclusion: Intestinal malrotation can manifest and present throughout adult life - as seen in this rare complication in the setting of another developmental defect. This case highlights the need for appropriate identification, embryological understanding and surgical management of problems such as these in the acute setting.

\section{Keywords}

Malrotation, Epigastric hernia, Hernia, Incarceration

\section{Case Description}

While uncommonly found pathologically together in the adult patient, visceral herniation and gut rotation go hand-in-hand during embryologic development. Rapid growth of the small bowel around week 6 of gestation results in physiologic herniation of the gut through the umbilical ring; the small bowel then rotates a full 270 degrees in a counter-clockwise direction to arrive at its normal anatomic position within the peritoneal cavity. Defects and missteps in these processes can have broad consequences for patients of all ages, as seen in our patient here.

A 40-year-old gentleman with a history of an epigastric hernia presented with two days of abdominal pain, discomfort, and a palpable lump in the anterior abdominal wall; his primary care doctor had promptly sent him to the Emergency Room after a brief office evaluation. The hernia had been reducible in months past, but the patient had not attempted reduction for several weeks. He denied any fevers, chills, nausea, vomiting, bowel changes or failure to move his bowels at that time. He also denied any previous surgeries. On physical examination, a tender, supra-umbilical hernia was noted with palpable loops of bowel present in hernia sac; the hernia was not-reducible even after abdominal relaxation and narcotics administration. No peritoneal signs were noted, and the abdomen was not distended. A CT scan obtained in the emergency room was consistent with colonic malrotation along the midline; the appendix and mesentery were also identified within the her- 
nia sac (Figure 1, Figure 2 and Figure 3). The neck of the defect measured approximately $3 \mathrm{~cm}$, and there was no evidence of appendicitis or obstruction at that time.

An exploratory laparotomy was performed with direct visualization of the appendix entering the ventral hernia sac. The sac was reduced, and an open appendectomy was performed; the rest of the bowel appeared viable and healthy. The malrotated colon was replaced in its proper orientation within the peritoneal cavity and the epigastric ventral defect was primarily repaired during closure of the laparotomy. No band ly-

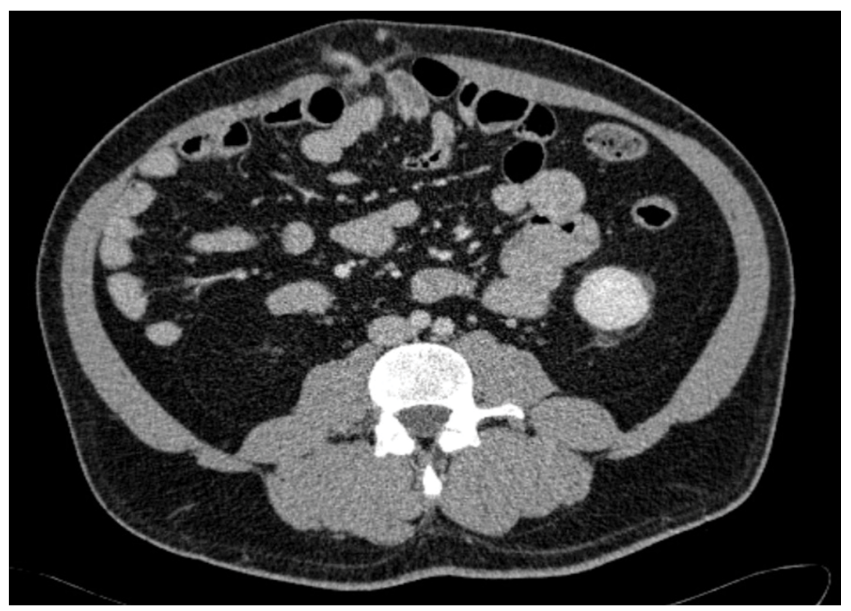

Figure 1: Axial CT of the malrotated colon along the midline with the appendix visualized entering the ventral hernia sac.

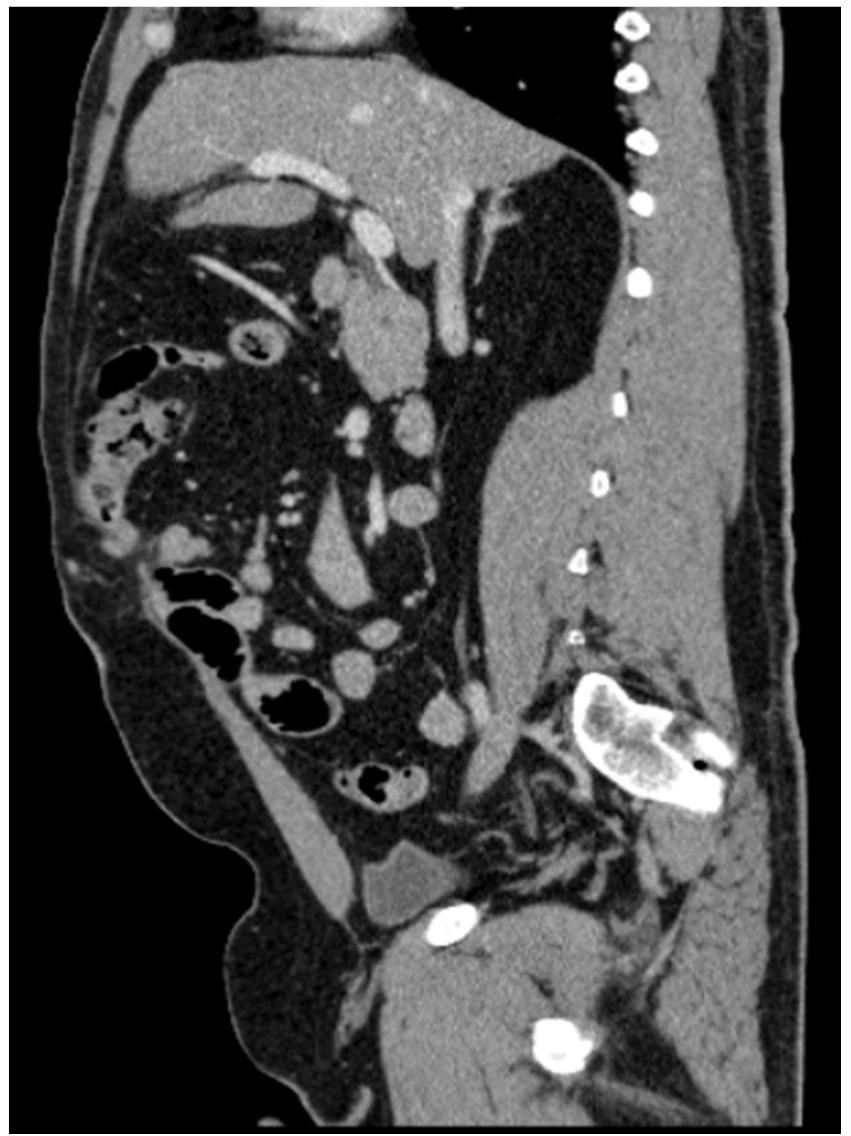

Figure 2: Sagittal view of the appendix entering the epigastric hernia sac. sis or cecopexy was performed. Pathologic analysis of the appendix was consistent with early inflammatory changes. The patient progressed as expected and was discharged the next day.

\section{Discussion}

Often a concern for the pediatric population, intestinal malrotation is considered a surgical emergency because of the risk of vascular compromise to the abdominal viscera. Clinical presentation with bilious emesis is almost always assumed to be malrotation until proven otherwise in children, and it frequently prompts rapid evaluation and intervention. The prevalence of malrotated guts within the general population is difficult to assess however, especially since not all present symptomatically; it is estimated that 1 case out of 6000 live births are identified out of clinical necessity, approximately $90 \%$ of which present during infancy [1]. The remaining $10 \%$ present in a variety of different ways in both childhood and adulthood. Malrotated patients beyond the neonatal period have been known to present with intestinal obstruction, chronic abdominal pain, peritonitis, malabsorption, biliary obstruction, delayed small bowel transit, and even midgut volvulus (A common presentation in neonates as well) $[2,3]$. Abdominal pain is the most common complaint that brings adults to medical attention, while the incidence of midgut volvulus (a serious concern in the neonatal period) declines with age [4]. Diagnosis in adults relies much more heavily on computed tomography for symptomatic malrotation rather than the contrast studies used in infants; serendipitous diagnosis intra-operatively is also a common occurrence. Surgical management in both adult and pediatric populations traditionally includes the Ladd's Procedure, or division of peritoneal bands, reduction of any gut volvulus, appendectomy and proper positioning of the gut with or without fixation within the peritoneum [5].

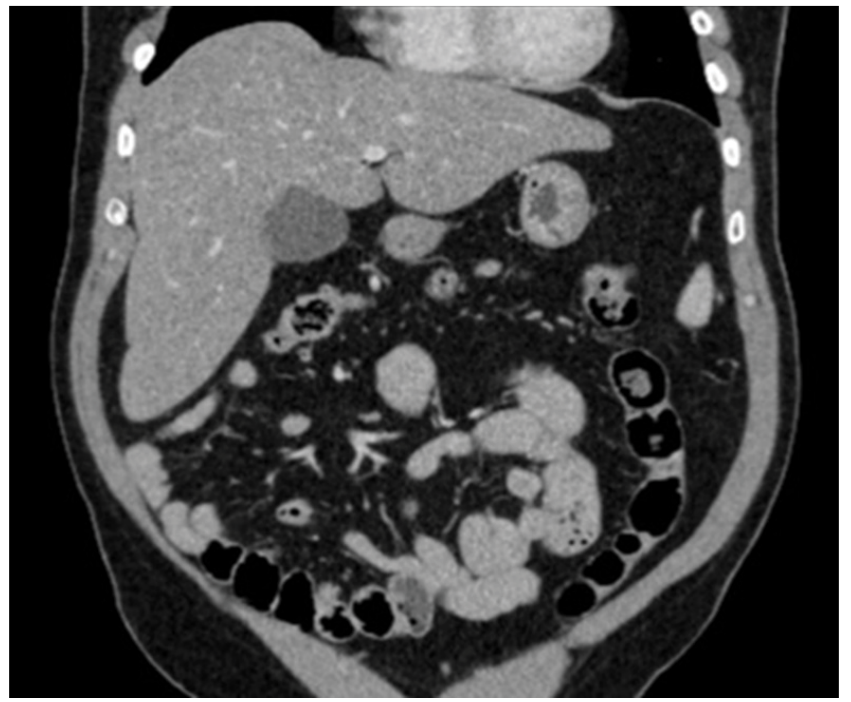

Figure 3: Coronal view of the malrotated colon in relation to the small bowel viscera/vasculature. 
Unlike malrotation, hernias can have both congenital and acquired etiologies. Epigastric hernias (herniations within the linea alba superior to the umbilicus) are found in as many as $5-10 \%$ of adults post-mortem, though not nearly as many present symptomatically (With pain, incarceration, strangulation, etc.) [6]. Their pathophysiologic origin is poorly understood, but theories include foci of fascial weakness around blood vessel entry points and inadequate fiber decussation within the linea alba [7]. Surgical incisions from both laparoscopy and laparotomy also increase the risk of herniation even in generally healthy patients. Their development is hastened by malnutrition and chronic corticosteroid therapy, along with mechanical and exertional forces. Patients typically present because of abdominal discomfort, pain, incarceration, or any part of the constellation of symptoms associated with bowel ischemia. Treatment can include laparotomy, open reduction and closure, laparoscopic repair, as well as the use of synthetic mesh.

The laparotomy for this patient was performed without difficulty; once the ventral defect had been identified through the incision, the appendix was properly reduced and excised for pathologic analysis. Adhesive bands were lysed. The classic Ladd's bands were not identified during the case, and no lysis of adhesions were required. The cecum itself was very mobile but was able to be placed in the proper anatomic position without cecopexy.

It is often difficult to identify patients with malrotation and their resultant sequelae until clinical presentation, though cases can be identified accidentally during the workup for unrelated concerns. Several attempts to define prognostic factors, including anatomical considerations have been presented in the literature. Some authors have suggested that patients with defects involving the duodenal/jejunal junction are more likely to suffer severe complications, including midgut volvulus, and therefore require preemptive correction [8]. A more measured approach, however, is one of caution and careful planning, with intervention for truly symptomatic patients and patients fully informed of all the risks and benefits involved. Ultimately, there are still no definitive criteria dictating when these patients would benefit most from an operation [9].

\section{Conclusion}

Intestinal malrotation, though typically a disease of the neonatal and pediatric population can present in a variety of ways and throughout one's lifespan. Management is dependent upon clinical presentation and acuity, though it is still unclear what role prophylactic intervention may play. We present a rare instance of appendiceal incarceration within an epigastric hernia, one of the many possible consequences associated with congenitally malrotated gut.

\section{Lessons Learned}

Adults presenting with sequelae of intestinal malrotation should undergo surgical management for acute issues, though it is unclear whether or not these patients would benefit from pre-emptive intervention in the setting of congenital malrotation. Co-existing conditions such as ventral hernias should be managed accordingly.

\section{Conflicts of Interest/Sources of Funding}

The authors declare no conflict of interest. The work was supported by the Department of Surgery at Temple University Hospital.

\section{References}

1. Puri P, Höllwarth ME (2009) Pediatric surgery: Diagnosis and management: Springer Science \& Business Media.

2. Spigland N, Brandt ML, Yazbeck S (1990) Malrotation presenting beyond the neonatal period. Journal of Pediatric Surgery 25: 1139-1142.

3. Powell DM, Biemann Othersen H, Smith CD (1989) Malrotation of the intestines in children: The effect of age on presentation and therapy. Journal of Pediatric Surgery 24: 777-780.

4. Nehra D, Goldstein AM (2011) Intestinal malrotation: Varied clinical presentation from infancy through adulthood. Surgery 149: 386-393.

5. Seymour NE, Andersen DK (2005) Laparoscopic Treatment of Intestinal Malrotation in Adults. Journal of the Society of Laparoendoscopic Surgeons 9: 298-301.

6. Courtney C, Lee A, Wilson C, O'Dwyer P (2003) Ventral hernia repair: a study of current practice. Hernia 7: 44-46.

7. Lang B, Lau H, Lee $F(2002)$ Epigastric hernia and its etiology. Hernia 6: 148-150.

8. Schey WL, Donaldson JS, Sty JR (1993) Malrotation of bowel: Variable patterns with different surgical considerations. Journal of Pediatric Surgery 28: 96-101.

9. Kapfer SA, Rappold JF (2004) Intestinal malrotation - not just the pediatric surgeon's problem. Journal of the American College of Surgeons 199: 628-635. 